\title{
ON POINCARÉ EXTENSIONS OF RATIONAL MAPS
}

\author{
CARLOS CABRERA, PETER MAKIENKO, AND GUILLERMO SIENRA
}

\begin{abstract}
There is a classical extension of Möbius automorphisms of the Riemann sphere into isometries of the hyperbolic space $\mathbb{H}^{3}$ which is called the Poincaré extension. In this paper, we construct extensions of rational maps on the Riemann sphere over endomorphisms of $\mathbb{H}^{3}$ exploiting the fact that any holomorphic covering between Riemann surfaces is Möbius for a suitable choice of coordinates. We show that these extensions define conformally natural homomorphisms on suitable subsemigroups of the semigroup of Blaschke maps. We extend the complex multiplication to a product in $\mathbb{H}^{3}$ that allows us to construct an extension of any given rational map which is right equivariant with respect to the action of $P S L(2, \mathbb{C})$.
\end{abstract}

\section{INTRODUCTION}

In the literature there are some constructions of extensions of rational dynamics from $\mathbb{C}$ to $\mathbb{H}^{3}$; see for example [9], 12] and [14. The constructions in [12] and [14] are based on Choquet's barycentric construction introduced and studied by A. Douady and C. Earle in [5]. Other important contributions on the barycentric constructions appear in [1] and 4.

As mentioned in the abstract, the basic idea of this paper is the following fact: "Any holomorphic covering between Riemann surfaces is a Möbius map on suitable coordinates." Then this covering can be extended to suitable Möbius manifolds. Let us discuss this idea in detail.

First, let us remind the reader that a Möbius $n$-orbifold is an $n$-orbifold endowed with an atlas such that the transition maps are Möbius transformations.

Given a discrete subgroup $\Gamma$ of Möbius transformations of the $n$-sphere $S^{n}$ acting properly discontinuous and freely on a domain $\Omega \subset S^{n}$, the quotient manifold $\Omega / \Gamma$ admits a Möbius structure. In the case when $n=3$, any manifold modeled on one of the following spaces $\mathbb{R}^{3}, S^{3}$, the unit ball $B^{3}$ in $\mathbb{R}^{3}, S^{2} \times \mathbb{R}$ or $B^{2} \times \mathbb{R}$ admits a Möbius structure; see [16].

Let $S_{1}$ and $S_{2}$ be two Möbius 2-orbifolds and let $R: S_{1} \rightarrow S_{2}$ be a finite degree covering which is Möbius on the respective Möbius structures. Assume that there exist two Kleinian groups $\Gamma_{1}$ and $\Gamma_{2}$ and two components $W_{1}$ and $W_{2}$ of the discontinuity sets $\Omega\left(\Gamma_{1}\right)$ and $\Omega\left(\Gamma_{2}\right)$ respectively, such that

$$
S_{i}=W_{i} / \operatorname{Stab}_{W_{i}}\left(\Gamma_{i}\right)
$$

for $i=1,2$. Now assume that there exists a Möbius map $\alpha(R): W_{1} \rightarrow W_{2}$ making

\footnotetext{
Received by the editors June 13, 2013 and, in revised form, November 10, 2014 and April 24,

2010 Mathematics Subject Classification. Primary 37F10, 37F30; Secondary 30F99.

This work was partially supported by PAPIIT IN-105912 and CONACYT CB2010/153850.
} 2015 . 
the following diagram commutative so that $\alpha(R)$ induces a homomorphism from $\Gamma_{1}$ to $\Gamma_{2}$ :

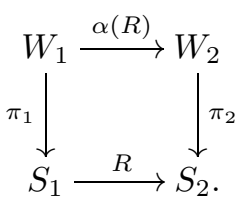

If

$$
M_{i}=\left(\overline{\mathbb{H}}^{3} \cup W_{i}\right) / \Gamma_{i},
$$

then $\alpha(R)$ induces a unique Möbius morphism

$$
\tilde{R}: M_{1} \rightarrow M_{2}
$$

which is an extension of $R: S_{1} \rightarrow S_{2}$. We call the map $\tilde{R}: M_{1} \rightarrow M_{2}$ a Poincaré extension of $R$. The map $\tilde{R}$ depends on the uniformizing groups $\Gamma_{1}$ and $\Gamma_{2}$. Hence, in general, for a given covering map $R$ there are many possibilities to construct a Poincaré extension. Note that the degree $\operatorname{deg}(\tilde{R})$ is equal to the index $\left[\Gamma_{2}\right.$ : $\left.\alpha(R) \circ \Gamma_{1} \circ \alpha(R)^{-1}\right]$. Hence $\operatorname{deg}(R) \leq \operatorname{deg}(\tilde{R})$ with equality when

$$
\operatorname{Stab}_{W_{i}}\left(\Gamma_{i}\right)=\Gamma_{i} .
$$

Given a Riemann surface $S$ with a fixed Möbius structure, in [6, sect.8] R. Kulkarni and U. Pinkal constructed a Möbius 3-manifold $M$ such that the surface $S$ is canonically contained in the boundary of $M$. If the structure of $S$ is uniformizable by a nontrivial Kleinian group then, this construction is given by the classical Poincaré extension of the uniformizing group and produces a complete hyperbolic manifold $M$. The construction is based on the following idea: Let $D$ be a round disk on $S$ with respect to the Möbius structure; that is, there exists a coordinate under which $D$ is a round disk in the plane. Using this coordinate, we attach a round half-ball in $\mathbb{H}^{3}$ to $D$. Then the 3 -manifold $M$ is the union of all the half open balls over all round disks in $S$.

On the Riemann sphere $\overline{\mathbb{C}}$ there is a unique complete Möbius structure $\sigma_{0}$, this is the standard Möbius structure on $\overline{\mathbb{C}}$. The construction of Kulkarni and Pinkal is clearer when $S$ is a planar surface with the standard Möbius structure.

When $S=\mathbb{C}^{*}$ with the standard Möbius structure, Kulkarni-Pinkal construction gives a canonical noncomplete Möbius 3-manifold which is Möbius equivalent to the 3-dimensional ball with the vertical diameter removed and endowed with the standard conformal structure on $B^{3}$. Now, consider a complete Möbius structure on $\mathbb{C}^{*}$. In this case, Kulkarni-Pinkal construction gives a complete hyperbolic 3manifold with the same underlying space as before. More generally, if $S=\overline{\mathbb{C}} \backslash F$, where $F$ is a closed set, then the Kulkarni-Pinkal extension $M$ is homeomorphic to $\mathbb{H}^{3} \backslash \operatorname{convhull}(F)$, where convhull $(F)$ is the hyperbolic convex hull in $\mathbb{H}^{3}$ of all points in $F$. The standard Möbius structure on $M$ is the extension of the standard Möbius structure on $S$.

The construction of Kulkarni and Pinkal motivates the idea of a model manifold for the Poincaré extension of a rational map. However, if $R$ is a branched selfcovering of $\overline{\mathbb{C}}$ and $\operatorname{deg}(R)>1$, then $R$ is not a Möbius covering with respect to the standard structure on any domain in $\overline{\mathbb{C}}$. We will restrict our attention to the case when $R$ is a rational map and $S_{1}$ and $S_{2}$ are two Riemannian orbifolds with underlying spaces contained in $\overline{\mathbb{C}}$, and such that $R: S_{1} \rightarrow S_{2}$ is a holomorphic 
covering. Let $\sigma_{2}$ be a uniformizable Möbius structure on $S_{2}$ and suppose that the pullback $\sigma_{1}:=R_{*}\left(\sigma_{2}\right)$ is also a uniformizable Möbius structure on $S_{1}$. If $\Gamma_{1}$ and $\Gamma_{2}$ are the uniformizing groups. Let $\tilde{R}$ be a Poincaré extension of $R$, such that $\Omega\left(\Gamma_{i}\right)$ are connected. Let $\phi_{i}: \partial M_{i} \rightarrow S_{i}$ be some identification maps and assume that there are homeomorphic extensions $\Phi_{i}: M_{i} \rightarrow \overline{\mathbb{H}}^{3}$ for each $\phi_{i}$. Then the map $\Phi_{2} \circ \tilde{R} \circ \Phi_{1}^{-1}$ is called geometric extension if and only if it satisfies the following conditions.

(1) The sets $\Phi_{i}\left(M_{i} \cup \partial M_{i}\right)$ are of the form $\overline{\mathbb{H}}^{3} \backslash\left\{\bigcup \gamma_{j}\right\}$ where each $\gamma_{j}$ is either a quasi-geodesic or a family of finitely many quasigeodesic rays with common starting point. There are no more than countably many curves $\gamma_{j}$. Here by quasigeodesic we mean the image of a hyperbolic geodesic by a quasiconformal automorphism.

(2) There exists a continuous extension, on all $\mathbb{H}^{3}$, which maps complementary quasi-geodesics to complementary quasigeodesics.

Hence, a geometric extension is an endomorphism of $\mathbb{H}^{3}$ such that its restriction to $\Phi_{1}\left(M_{1}\right)$ is a Poincaré extension.

Remark. In a Poincaré extension construction the domain and the range are, in principle, different, and hence we loose dynamics. This explains why we introduce the notion of geometric extension. So, we have an endomorphism of $\overline{\mathbb{H}}^{3}$ whose restriction to the boundary is the given rational map $R$.

A geometric extension is unique up to the bi-action of a group $G$ of automorphisms of $\overline{\mathbb{H}}^{3}$ which acts as the identity on $\partial \mathbb{H}^{3}$. However, different geometric extensions may have different dynamical behavior.

Let $\operatorname{Rat}_{d}(\mathbb{C})$ denote the set of rational maps $R$ of degree $d$. Let $A \subset \operatorname{Rat}_{d}(\mathbb{C})$. Assume that there exist a map

$$
\operatorname{Ext}: A \rightarrow \operatorname{End}\left(\overline{\mathbb{H}}^{3}\right)
$$

such that $\operatorname{Ext}(R)$ is an extension of $R$ for every $R$ in $A$. Then for every pair of maps $h, g$ in the Möbius group $M o b$ we define

$$
\widetilde{\operatorname{Ext}}(g \circ R \circ h)=\hat{g} \circ \operatorname{Ext}(R) \circ \hat{h}
$$

where $\hat{h}$ and $\hat{g}$ are the classical Poincaré extensions of the maps $h$ and $g$ in the hyperbolic space, respectively.

If $\widetilde{E x t}$ is a well-defined map from the Möbius bi-orbit of $A$ to $\operatorname{End}\left(\overline{\mathbb{H}}^{3}\right)$, then we call Ext a conformally natural extension of $A$.

In particular, when the bi-action of $\operatorname{PSL}(2, \mathbb{C})$ on $A$ has no fixed points, any map Ext : $A \rightarrow \operatorname{End}\left(\mathbb{H}^{3}\right)$ defines a map $\widetilde{E x t}$ on the Möbius bi-orbit which is a conformal natural extension. If the action has fixed points then, in order to obtain a conformally natural extension, the map Ext has to be consistent with the Möbius action. The situation is tricky even in the case when $A$ consists of a single point $R$.

Let $\hat{R}$ be a geometric extension of a rational map $R$, then any rational map on the Möbius bi-orbit of $R$ has a geometric extension. Namely, if $h$ and $g$ are elements in $P S L(2, \mathbb{C})$, then $Q=g \circ R \circ h$ has a geometric extension with the same uniformizing groups $\Gamma_{1}$ and $\Gamma_{2}$, the projections $p_{1}=\pi_{1} \circ \hat{h}$ and $p_{2}=\pi_{2} \circ \hat{g}^{-1}$ and the associated manifolds are $N_{1}=\hat{h}^{-1}\left(M_{1}\right)$ and $N_{2}=\hat{g}\left(M_{2}\right)$. We define an extension of $Q$ by the formula $\hat{Q}=\hat{g} \circ \hat{R} \circ \hat{h}$. 
However, one should be careful in the situation when, for a given $R$, there are other elements $g^{\prime}$ and $h^{\prime}$ in $P S L(2, \mathbb{C})$ such that $Q=g^{\prime} \circ R \circ h^{\prime}$. This situation happens when there are elements $h_{1}$ and $h_{2}$ in $P S L(2, \mathbb{C})$ such that

$$
R \circ h_{2}=h_{1} \circ R \text {. }
$$

If there are no such $h_{1}$ and $h_{2}$, then any geometric extension of $R$ is conformally natural. However, if such elements do exist but the Poincaré extensions of $h_{i}$ are Möbius automorphisms of $M_{i}$ with respect to their Möbius structures, then the extension $R \mapsto \hat{R}$ is conformally natural.

In this article, we will investigate the existence of extensions of $R$, defined in the hyperbolic space $\mathbb{H}^{3}$, satisfying as many as possible of the following desirable conditions.

(1) Geometric. As defined above.

(2) Same degree. The index $\left[\Gamma_{2}: \Gamma_{1}\right]$ is equal to the degree of the map $R$.

(3) Dynamical. Let $\hat{R}$ be a Poincaré extension such that $M_{1}=M_{2}$, then $\hat{R}$ is dynamical. In particular, these are extensions $\operatorname{Ext}$ such that $\operatorname{Ext}\left(R^{n}\right)=$ $\operatorname{Ext}(R)^{n}$ for $n=1,2, \ldots$

(4) Semigroup homomorphisms. A stronger version of the previous property is to find semigroups $\mathcal{S}$, of rational maps, for which there is an extension Ext defined in all $\mathcal{S}$ such that $\operatorname{Ext}(R \circ Q)=\operatorname{Ext}(R) \circ \operatorname{Ext}(Q)$.

(5) Equivariance under Möbius actions. We look for conformally natural extensions of subsets $A$ of $\operatorname{Rat}_{d}(\mathbb{C})$.

Assume that $R$ has a geometric extension and let $\Gamma_{2}$ be the group that uniformizes the Möbius structure on $S_{2}$. Then the discontinuity set $\Omega\left(\Gamma_{2}\right)$ consists of the orbit of a unique component $C$. The stabilizer of $C$ uniformizes the surface $S_{2}$. In this case the group $\Gamma_{2}$ is either totally degenerated of Schottky type or of Web group type. When $\Gamma_{2}$ is a web group, the orbit of the component $C$ is infinite. Thus, in general, it is possible that condition (2) may not be satisfied.

On the other hand, if the component $C$ uniformizing the surface $S_{2}$ is invariant under $\Gamma_{2}$, then condition (2) is satisfied. For this reason, we restrict our discussion to this case. A group having an invariant component is called a function group. By Maskit's theorem any function group can be represented as a Klein-Maskit combination of the following groups:

- Totally degenerated groups.

- Groups of Schottky type.

According to this list of groups we call a geometric extension totally degenerated, or of Schottky type, whenever the uniformizing group has the corresponding property. Totally degenerated groups appear as geometric limits of quasifuchsian groups. In fact, totally degenerated groups belong to the boundary of a Bers slice.

This paper is organized as follows.

In Section 2 we will discuss Hurwitz spaces and quasifuchsian extensions. We will construct an extension, the radial extension, that satisfies (2), (3) and (4) in the previous list. We will give some conditions for which the radial extension is geometric.

Section 3 is devoted to Schottky type extensions.

In Section 4 we discuss the construction of a right equivariant extension, with respect to the action of $P S L(2, \mathbb{C})$, of all rational maps which is connected to a product structure defined on the hyperbolic space. 
Finally, in Section 5, we discuss examples of extensions and surgeries of Maskit type.

\section{Fuchsian Structures, DEgenerated And RAdial EXtensions}

Given a rational map $R$, let $C V(R)$ denote the critical values of $R$ and take $S_{2}=\overline{\mathbb{C}} \backslash C V(R)$ and $S_{1}=R^{-1}\left(S_{2}\right)$, then $R: S_{1} \rightarrow S_{2}$ is a covering. We assume that the set of critical values of $R$ contains at least three points, say $b_{1}, b_{2}$ and $b_{3}$, so that $S_{2}$ is a hyperbolic Riemann surface. In order to get normalized maps we pick three points $a_{1}, a_{2}$ and $a_{3}$ in $\overline{\mathbb{C}}$ such that $R\left(a_{i}\right)=b_{i}$ for $i=1,2,3$.

We say that two branched coverings $R$ and $Q$, of the Riemann sphere onto itself, are Hurwitz equivalent if there are quasiconformal homeomorphisms $\phi$ and $\psi$, making the following diagram commutative:

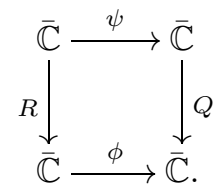

Given a rational map $R$, the Hurwitz space $H(R)$ is the set of all rational maps $Q$ that are Hurwitz equivalent to $R$. The topology we are considering on $H(R)$ is the compact open topology.

Let $f: S_{2} \rightarrow S_{2}^{\prime}$ be a representative of a point in the Teichmüller space $T\left(S_{2}\right)$ with Beltrami coefficient $\mu$ and fixing the points $b_{i}$. Let $R_{*}(\mu)$ be the pullback of $\mu$ under $R$. Let $h_{f}$ be the solution defined on the Riemann sphere of the Beltrami equation for the coefficient $R_{*}(\mu)$ and take $S_{1}^{\prime}=h_{f}\left(S_{1}\right)$. Let us define the map $\tau: T\left(S_{2}\right) \rightarrow H(R)$ so that $\tau(f)$ is the rational map making the following diagram commutative:

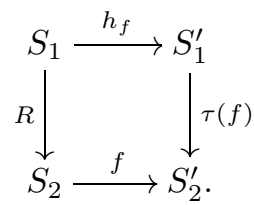

The map $\tau$ is well defined and continuous, since the solution of the Beltrami equation depends analytically on $\mu$. We call the space $H_{\tau}(R)=\tau\left(T\left(S_{2}\right)\right)$ the reduced Hurwitz space of $R$. The closure of the bi-orbit by the Möbius group of $H_{\tau}(R)$ is the whole of $H(R)$. The group $P S L(2, \mathbb{C})$ also acts by conjugation on $H(R)$. The space of orbits by conjugation fibers over the reduced Hurwitz space $H_{\tau}(R)$.

Let $f$ be an element of the Mapping Class Group $M C G\left(S_{2}\right)$ such that $f\left(b_{i}\right)=b_{i}$. We say that $f$ is liftable with respect to $R$ if there exists a map $g: S_{1} \rightarrow S_{1}$, such that $g\left(a_{i}\right)=a_{i}$ and makes the following diagram commutative:

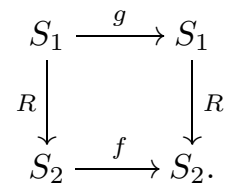

In this case, we say that $g$ is the lifted map of $f$ with respect to $R$. Let $\mathcal{G}$ be the subgroup of the mapping class group $M C G\left(S_{2}\right)$ which consists of all liftable 
elements with respect to $R$. We identify $H_{\tau}(R)$ with the space $T_{2}\left(S_{2}\right) / \mathcal{G}$ as the following theorem suggests.

Theorem 1. The space $H_{\tau}(R)$ is homeomorphic to $T\left(S_{2}\right) / \mathcal{G}$.

Proof. The map $\tau$ induces a continuous map $\tilde{\tau}$ from $T\left(S_{2}\right) / \mathcal{G}$ onto $H_{\tau}(R)$. Now we will show that $\tau\left(\phi_{1}\right)=\tau\left(\phi_{2}\right)$ if and only if $\phi_{2}^{-1} \circ \phi_{1} \in \mathcal{G}$.

Let $f=\phi_{2}^{-1} \circ \phi_{1}$, and assume that $f$ belongs to $\mathcal{G}$ and let $g$ be the lifted map of $f$ with respect to $R$, so if $h_{\phi_{1}}$ and $h_{\phi_{2}}$ are the maps associated with $\tau\left(\phi_{1}\right)$ and $\tau\left(\phi_{2}\right)$ respectively, we have $h_{\phi_{2}} \circ g=h_{\phi_{1}}$ by the normalization of $g$; hence

$$
\phi_{2} \circ f \circ R=\phi_{1} \circ R=\tau\left(\phi_{1}\right) \circ h_{\phi_{1}} .
$$

On the other hand,

$$
\phi_{2} \circ f \circ R=\phi_{2} \circ R \circ g=\tau\left(\phi_{2}\right) \circ h_{\phi_{2}} \circ g,
$$

so we get

$$
\tau\left(\phi_{1}\right)=\tau\left(\phi_{2}\right)
$$

Reciprocally, if $\tau\left(\phi_{1}\right)=\tau\left(\phi_{2}\right)$, then $f=\phi_{2}^{-1} \circ \phi_{1}$ fixes the points $b_{i}$. Since $h_{\phi_{2}}^{-1} \circ h_{\phi_{1}}$ is a lift of $f$ with respect to $R$, the map $f$ belongs to $\mathcal{G}$.

Since $T\left(S_{2}\right) / \mathcal{G}$ is metrizable and of finite dimension, we can take a closed and bounded ball with respect to a metric $D$. Since $H_{\tau}(R)$ is a Hausdorff space then the restriction of $\tilde{\tau}$ on $D$ is a homeomorphism. Hence $\tilde{\tau}$ itself is a homeomorphism.

The following theorem gives a description of compact subsets of $H(R)$. For a quasiconformal map $f$, let $K_{f}(z)$ be the distortion of $f$ at the point $z$.

Theorem 2. If $\left\{f_{i}\right\}$ is a family of quasiconformal maps on the sphere $\overline{\mathbb{C}}$ fixing the points $b_{1}, b_{2}$ and $b_{3}$. Let

$$
A_{n}=\left\{z: K_{f_{i}}(z) \geq n \text { for } i \text { big enough }\right\} .
$$

Assume that $A_{\infty}=\overline{\bigcap A_{n}}$ is a compact subset of $S_{2}$. So we have:

- If $A_{\infty}$ does not separate the critical values of $R$, then the family $\left\{\left[f_{i}\right]\right\}$ is bounded in $T\left(S_{2}\right)$.

- If there exist a domain $D_{0}$ contained in $S_{2} \backslash A_{\infty}$ such that $D_{0}$ contains at least two of the points $b_{i}$ and $W_{0}=R^{-1}\left(D_{0}\right)$ is connected, then the respective classes $\left\{\tau\left(\left[f_{i}\right]\right)\right\}$ are bounded in $\operatorname{Rat}_{d}(\mathbb{C})$.

Proof. For the first item, let $U$ be a neighborhood of $A_{\infty}$ that is compactly contained in $S_{2}$, does not separate $S_{2}$ and such that every component of $U$ is simply connected with analytic boundary.

The restrictions of $f_{i}$ on $\partial U$ are quasisymmetric maps with uniform bound of distortion. Using Douady-Earle extension, the maps $\left.f_{i}\right|_{\partial U}$ extend to maps $\tilde{f}_{i}$, defined on the interior of $U$, with uniformly bounded distortion. Since $f_{i}$ and $\tilde{f}_{i}$ have the same values on the boundary, the maps $f_{i}$ and $\tilde{f}_{i}$ are homotopic, and define the same points in $T\left(S_{2}\right)$. Hence the family $f_{i}$ have uniformly bounded Beltrami coefficients, so defines a bounded set in $T\left(S_{2}\right)$.

Since $\operatorname{Rat}_{d}(\mathbb{C})$ can be identified with an open and dense subset of the projective space, it is enough to prove that all the limit maps of $\left\{\tau\left(f_{i}\right)\right\}$ are rational maps of the same degree. Let $g_{i}$ be quasiconformal automorphisms of $\overline{\mathbb{C}}$ fixing the points $a_{i}$ and such that $f_{i} \circ R=\tau\left(f_{i}\right) \circ g_{i}$. Under the conditions of the second item, the accumulation functions of the families $\left\{\left.f_{i}\right|_{D}\right\}$ and $\left\{\left.g_{i}\right|_{W_{0}}\right\}$ are nonconstant 
quasiconformal functions. Let $R_{\infty}$ be a rational map which is an accumulation point of the maps $\tau\left(f_{i}\right)$, then there exist two nonconstant quasiconformal functions $f_{\infty}$ and $g_{\infty}$ and domains $O=h_{\infty}(D)$ and $X=g_{\infty}^{-1}\left(W_{0}\right)$ on which $f_{\infty} \circ R=R_{\infty} \circ g_{\infty}$. Then $\operatorname{deg}\left(R_{\infty \mid O}\right)=\operatorname{deg}\left(R_{\mid W_{0}}\right)=\operatorname{deg}(R)$, as we wanted to prove.

2.1. Bers slices. Let $\Delta=\{z:|z|<1\}$ and $\Delta^{*}=\{z:|z|>1\}$. We denote the boundary of $\Delta$ by $\mathbb{S}^{1}$. Now let us consider again the rational map $R$ and surfaces $S_{i}$. Let $\Gamma_{i}$ be Fuchsian groups that uniformizes the surfaces $S_{i}$. By the Monodromy Theorem there exist a Möbius map $\alpha$ such that $\tilde{\Gamma}=\alpha \Gamma_{1} \alpha^{-1}$ is a subgroup of $\Gamma_{2}$. If $p: \Delta \rightarrow S_{1} \cong \Delta / \tilde{\Gamma}$ is the orbit projection. Then defining $\pi_{1}=p \circ \alpha^{-1}$ gives the following diagram:

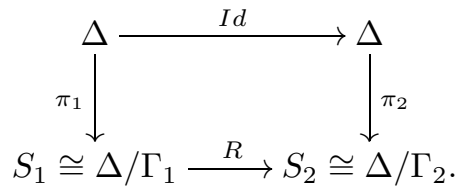

Moreover, $\left[\Gamma_{2}: \Gamma_{1}\right]=\operatorname{deg}(R)$ since $R$ is a covering. We call the pair $\left(\Gamma_{1}, \Gamma_{2}\right)$ a uniformization of $R$. Simultaneously we have uniformization of the surfaces $S_{i}^{*}=$ $\Delta^{*} / \Gamma_{i}$ and the map $Q: S_{1}^{*} \rightarrow S_{2}^{*}$ given by $Q(z):=\overline{R(\bar{z})}$. Now these groups are acting on the complement of the unit disk $\Delta^{*}$.

Let $D\left(\Gamma_{2}\right)$ be the space of groups $\Gamma$ such that there exist a quasiconformal map $f$ such that $\Gamma=f \circ \Gamma_{2} \circ f^{-1}$ and such that the Beltrami differential $\mu_{f}$ is equal to 0 in $\Delta^{*}$. Now put

$$
\operatorname{Def}\left(\Gamma_{2}\right)=D\left(\Gamma_{2}\right) / P S L(2, \mathbb{C}) .
$$

Analogously, we define $D e f^{*}\left(\Gamma_{2}\right)$ as the space of deformations of $\Gamma_{2}$ on $\Delta^{*}$. By Bers Theorem, both spaces $\operatorname{Def}\left(\Gamma_{2}\right)$ and $\operatorname{De} f^{*}\left(\Gamma_{2}\right)$ have compact closure on the space of classes of faithful and discrete representations

$$
\Gamma_{2} \hookrightarrow P S L(2, \mathbb{C}) .
$$

These closures of $\operatorname{Def}\left(\Gamma_{2}\right)$ and $\operatorname{Def} f^{*}\left(\Gamma_{2}\right)$ are called Bers slices of the Teichmüller space. In these cases, the Bers slices consist of function groups with a simply connected invariant component. Geometrically finite groups contained in the Bers slice are either quasifuchsian or cusps. Definitions and properties can be found in the papers by Bers [3], by Maskit [10] and by McMullen [11. If a group $G$ in the boundary of the Bers slice has a connected region of discontinuity, then $G$ is totally degenerated. By theorems of Bers, Maskit and McMullen (see [3], [10] and 11]), totally degenerated groups and cusps are both dense on the boundary of the Bers slice.

Any group $G$ in the Bers slice defines a 3-hyperbolic manifold $M(G)$ with boundary. Given a uniformization $\left(\Gamma_{1}, \Gamma_{2}\right)$ of $R$, let us consider $M_{1}:=M\left(\Gamma_{1}\right)$ and $M_{2}:=M\left(\Gamma_{2}\right)$ the associated 3-hyperbolic manifolds with boundary. Then the inclusion $\Gamma_{1}$ in $\Gamma_{2}$ defines a Möbius map

$$
F: M_{1} \rightarrow M_{2} \text {. }
$$

The restriction of $F$ to the boundary components of $M_{1}$ define maps which are rational in coordinates. Let $\Sigma_{i} \subset \partial M_{i}$ be the respective invariant components of $\Gamma_{i}$. Then the map $F: \Sigma_{1} \rightarrow \Sigma_{2}$ belongs either to the Hurwitz space $H(R)$ or to $H(Q)$. 
If a group $G$ in the Bers slice $\operatorname{Def}\left(\Gamma_{2}\right)$ is geometrically finite, then $G$ is a cusp or quasifuchsian. In those situations there is a boundary component $S$ in $M(G)$ conformally equivalent to $\Delta^{*} / \Gamma_{2}$. In the case that there are cusps on the group, we regard the set of all components as a connected surface with nodes $\tilde{S}$.

Question. Assume that $G_{i}$ converges, in the Bers slice, to a totally degenerated group $G$. Is it true that the accumulation set of the associated rational maps may contain constants maps?

Now we are ready to prove the main result of this section. Let us begin with the following definition. Let $G$ be a totally degenerated group. Then we call the group $G$ acceptable for the rational map $R$ if and only if the following conditions hold:

- There are two uniformizable Möbius orbifolds $S_{i}$ supported on the Riemann sphere, such that $R: S_{1} \rightarrow S_{2}$ is a holomorphic covering.

- If $\Gamma_{2}$ is a Fuchsian group uniformizing $S_{2}$, then $G$ belongs to $D e f^{*}\left(\Gamma_{2}\right)$.

- The manifold $M(G)$ is homeomorphic to $\partial M(G) \times \mathbb{R}_{+}$, where $\mathbb{R}_{+}$denotes the set of nonnegative real numbers.

Let $\pi: \mathbb{H}^{3} \cup \Omega(G) \rightarrow M(G)$ be the orbit projection. Under the homeomorphism of the last item, let us define, for every $t$ in $\mathbb{R}_{+}$the set $\Omega(G)_{t}=\pi^{-1}(\partial M \times t)$. Hence, the space $\mathbb{H}^{3} \cup \Omega(G)$ is foliated by the sets $\Omega(G)_{t}$ and there exists a continuous family of homeomorphisms $f_{t}: \Omega(G) \rightarrow \Omega(G)_{t}$ which commutes with $G$ and $f_{0}=I d$.

Let $\mathbb{B}^{3}$ denote the unit ball model for the hyperbolic space. Given a rational map $R$, we define the radial extension $\tilde{R}$ as follows. For every $\lambda \in[0,1]$ and $(x, y, z) \in \mathbb{R}^{3}$, let $H_{\lambda}(x, y, z)=(\lambda x, \lambda y, \lambda z)$. Then we have

$$
\overline{\mathbb{B}}^{3}=\bigcup_{\lambda \in[0,1]} H_{\lambda}\left(\partial \mathbb{B}^{3}\right) .
$$

Now define $\hat{R}(0,0,0)=(0,0,0)$ and for $v \in \overline{\mathbb{B}}^{3}$, different from 0 , define $\hat{R}(v)=$ $H_{\|v\|} \circ R \circ H_{\|v\|}^{-1}$ where $\|v\|$ denotes the euclidean distance to $v$ from the origin.

Theorem 3. If there exist an acceptable group for $R$ then the radial extension of $R$ is geometric.

Proof. Let $G_{2}$ be an acceptable group for $R$, then there exists $\phi: \Omega\left(G_{2}\right) \rightarrow \Delta$ such that it induces an isomorphism $\phi_{*}: G_{2} \rightarrow \Gamma_{2}$, then $G_{2}$ has a finite index subgroup $G_{1}=\phi_{*}^{-1}\left(\Gamma_{1}\right)$, such that the map $\alpha(R): M\left(G_{1}\right) \rightarrow M\left(G_{2}\right)$ is Möbius. Moreover, the manifold $M\left(G_{1}\right)$ is a manifold homeomorphic to $\partial M\left(G_{1}\right) \times \mathbb{R}_{+}$. We have to show that the radial extension is equivalent to $\alpha(R)$, so it is geometric. Again, each $M\left(G_{i}\right)$ is homeomorphic to $S_{i} \times \mathbb{R}_{+}$, and the horizontal foliation in $M\left(G_{1}\right)$ is the pullback by $F: M\left(G_{1}\right) \rightarrow M\left(G_{2}\right)$ of the horizontal foliation in $M\left(G_{2}\right)$. Hence, there exist a covering $\phi: S_{1} \times \mathbb{R}_{+} \rightarrow S_{2} \times \mathbb{R}_{+}$and homeomorphisms $h_{1}, h_{2}$ such that the following diagram commutes:

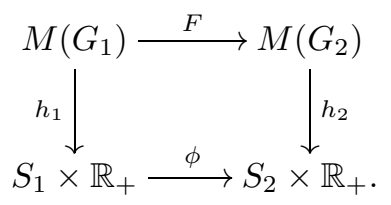


Hence, $\phi(x)=F(x)=R(x)$ for $x$ in $S_{1}$. There are two families of homeomorphisms $\psi_{t}$ and $\chi_{t}$ such that

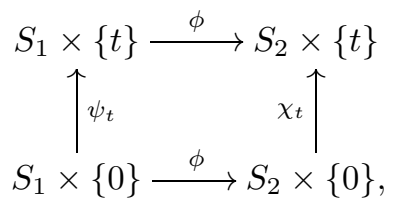

so that $\phi$ preserves the parameter $t$. Now consider a homeomorphism $k:[0, \infty] \rightarrow$ $[0,1]$ such that $k(0)=1$ and $k(\infty)=0$. For $i=0,1$, let us identify the sets $S_{i} \times 0$ with the corresponding $S_{i}$ on the Riemann sphere. Hence, we define two homeomorphisms $\psi$ and $\chi$ such that $\psi(x, t)=H_{k(t)}\left(\psi_{t}^{-1}(x)\right)$ and $\chi(x, t)=H_{k(t)}\left(\chi_{t}^{-1}(x)\right)$, where $H_{t}(x, y, z)=(t x, t y, t z)$. Since $\psi$ and $\chi$ are the identity on the boundary, these homeomorphisms uniformize the extension of $\phi$ over $S_{1}$ and $S_{2}$.

We have not found a reference that shows that the manifold of a totally degenerated group in the Bers slice of a finitely generated group is always a product. However, Michael Kapovich kindly gave us arguments to show that this happens. The arguments are based upon a work of Waldhausen and the solution of the Tame Conjecture.

\section{SChOtTKY TyPE EXTENSIONS OF RATIONAL MAPS}

In this section, we prove that any map in the Hurwitz space of a Blaschke map has an extension of Schottky type that satisfies the properties (1), (2) and (3) in the introduction. We also construct an extension of Blaschke maps that satisfies almost all five conditions: this extension does not satisfy condition (4). However, if we take the group of Möbius transformations preserving the unit circle instead of $\operatorname{PSL}(2, \mathbb{C})$, then the modified condition (4) holds.

A Blaschke map $B: \overline{\mathbb{C}} \rightarrow \overline{\mathbb{C}}$ is a rational map that leaves the unit disk $\Delta$ invariant. If $d$ is the degree of $B$, then there exist $\theta \in[0,2 \pi]$ and $d$ points $\left\{a_{1}, \ldots, a_{d}\right\}$ in $\Delta$ such that

$$
B(z)=e^{i \theta}\left(\frac{z-a_{1}}{1-\bar{a}_{1} z}\right) \ldots\left(\frac{z-a_{d}}{1-\bar{a}_{d} z}\right)
$$

Let us denote by $B_{1}=\left.B\right|_{\Delta}$ and $B_{2}=\left.B\right|_{\Delta^{*}}$, then $B_{2}(z)=\frac{1}{\bar{z}} \circ B_{1}(z) \circ \frac{1}{\bar{z}}$.

Let $C V(B)$ be the set of critical values of $B$, then define $S_{2}=\overline{\mathbb{C}} \backslash C V(B)$ and $S_{1}=B^{-1}\left(S_{2}\right)$. Thus $B: S_{1} \rightarrow S_{2}$ is a holomorphic covering and the surfaces $S_{i}$ are symmetric with respect to $\mathbb{S}^{1}$.

The class of Blaschke maps allows us to build a specific topological construction based upon Schottky coverings of Riemann surfaces. What is special about Blaschke maps is that every Blaschke map commutes with the involution $\tau(z)=\frac{1}{\bar{z}}$. Recall that Fuchsian groups of second type are Schottky type Fuchsian groups.

Theorem 4. Given a Blaschke map $B$, such that $B: S_{1} \rightarrow S_{2}$ is a covering and the surfaces $S_{i}$ are symmetric surfaces with respect to $\mathbb{S}^{1}$. There are two Fuchsian groups of second type, $\Gamma_{1}$ and $\Gamma_{2}$ such that $\Omega\left(\Gamma_{i}\right) / \Gamma_{i}=S_{i}$, where $\Omega\left(\Gamma_{i}\right)$ is the 
discontinuity set of $\Gamma_{i}$ for $i=1,2$. Furthermore, there exist a Möbius map $\alpha$ : $\Omega\left(\Gamma_{1}\right) \rightarrow \Omega\left(\Gamma_{2}\right)$ making the following diagram commutative:

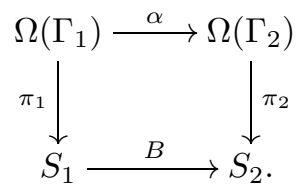

Also $\left[\Gamma_{2}: \alpha \Gamma_{1} \alpha^{-1}\right]=\operatorname{deg}(B)$.

Proof. For $i=1,2$ let $\Delta_{i}=\bar{\Delta} \cap S_{i}$ and $\Delta_{i}^{*}=\bar{\Delta}^{*} \cap S_{i}$.

The Simultaneous Uniformization Theorem of Bers [2], ensures that there exist a Fuchsian group $\Gamma_{2}$ acting in $\overline{\mathbb{C}}$, with $\Delta / \Gamma_{2}=\Delta_{2}$ and $\Delta^{*} / \Gamma_{2}=\Delta_{2}^{*}$. The limit set $\Lambda\left(\Gamma_{2}\right)$ is contained in $\mathbb{S}^{1}$.

Similarly, there is a Fuchsian group $\Gamma_{1}$ such that $\Delta^{*} / \Gamma_{1}=\Delta_{1}^{*}$ and $\Delta / \Gamma_{1}=\Delta_{1}$ and $\Lambda\left(\Gamma_{1}\right) \subset \mathbb{S}^{1}$.

The map $B_{i}$ lifts to Möbius maps $\alpha_{1}: \Delta \rightarrow \Delta$ and $\alpha_{2}: \Delta^{*} \rightarrow \Delta^{*}$. Moreover, since $\Omega_{2}$ is a Riemann surface anti-conformally equivalent to $\Omega_{1}$, we can choose $\alpha_{2}$ such that $\alpha_{2}(z)=\frac{1}{\bar{z}} \circ \alpha_{1} \circ \frac{1}{\bar{z}}$ and these maps agree at $\mathbb{S}^{1} \backslash \Lambda(G)$. Being that $\Lambda(G)$ is a Cantor set, then the map $\alpha: \overline{\mathbb{C}} \backslash \Lambda(G) \rightarrow \overline{\mathbb{C}} \backslash \Lambda(G)$ defined as $\alpha \mid \Delta_{i}=\alpha_{i}$ extends to a Möbius map $\alpha$ defined on the Riemann sphere.

By Theorem 4, a Blaschke map admits a Poincaré extension which follows from the diagram below:

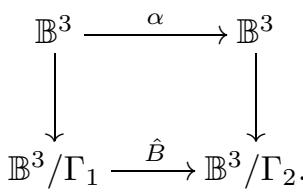

Let us observe that $\Gamma_{1}$ and $\Gamma_{2}$ are Schottky type groups with parabolic generators. Hence $\mathbb{B}^{3} / \Gamma_{1}$ and $\mathbb{B}^{3} / \Gamma_{2}$ are homeomorphic to the complement in $\mathbb{B}^{3}$ of a finite number of geodesics connecting symmetric perforations of the surfaces $S_{i}$.

The arguments in Theorem 4 work in a more general situation. The key facts are Bers Simultaneous Uniformization Theorem and the symmetry of respective orbifolds. So we have the following.

Corollary 5. Let $W_{1}$ and $W_{2}$ be any two given connected symmetric orbifolds supported on the Riemann sphere. If $B: W_{1} \rightarrow W_{2}$ is a covering symmetric with respect to $\mathbb{S}^{1}$, then the conclusion of Theorem 4 holds for $B$.

Let $G \mathcal{O}(P(B))$ be the grand orbit of the postcritical set $P(B)$ and take $S=$ $\overline{\mathbb{C}} \backslash \overline{G \mathcal{O}(P(B))}$. When $S$ is a symmetric surface such that $D=S \cap \Delta$ and $D^{*}=S \cap \Delta^{*}$ are connected, then $B: S \rightarrow S$ is a holomorphic self-covering, and we have the following corollary. 
Corollary 6. There exist a Fuchsian group $\Gamma$ and $\alpha$ in $P S L(2, \mathbb{R})$ such that $\alpha_{*}(\Gamma)=\alpha \Gamma \alpha^{-1}$ is a subgroup of $\Gamma$. Moreover, we have that $\Omega(\Gamma)=\Delta \cup \Delta^{*}$, $\Delta^{*} / \Gamma=D^{*}$, and $\Delta / \Gamma=D$. Finally, for every $n$ the following diagram is commutative:

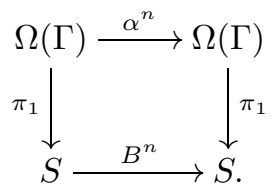

Proof. The proof is essentially the same as in Theorem 4 using the symmetry of the surfaces plus the fact that $B$ defines a self-covering of the surface $S$.

As noted after Theorem 4 we have that the Poincaré extension of $B$ is an endomorphism of $\mathbb{B}^{3} / \Gamma$, so this Poincaré extension is dynamical.

Remark. By the classification of periodic Fatou components, $D$ and $D^{*}$ are disconnected if and only if the postcritical set is infinite and there exist fixed critical points in $D$ and $D^{*}$, respectively. In other words, $D$ and $D^{*}$ are connected for an open and everywhere dense set in the space of Blaschke maps of any given degree. In particular, $D$ and $D^{*}$ are connected for every Blaschke map of degree 2 .

Again, the argument in the corollary above can be generalized as in the following corollary:

Corollary 7. Given a Blaschke map B, let $A$ be a completely invariant symmetric closed subset of $\overline{\mathbb{C}}$ which contains all critical points of $B$. Let $W=\overline{\mathbb{C}} \backslash A$ and assume that $U=W \cap \Delta$ and $U^{*}=W \cap \Delta^{*}$ are connected. Then $\left\{B^{n}: U^{*} \rightarrow U^{*}\right\}$ and $\left\{B^{n}: U \rightarrow U\right\}$ are semigroups of coverings and the Poincaré extensions of these semigroups are dynamical.

Now let us consider the case of decomposable Blaschke maps. Assume that $B=B_{1} \circ B_{2}$ where $B_{1}$ and $B_{2}$ are Blaschke maps. Then, if $Q=B_{2} \circ B_{1}$, there are semiconjugacies:

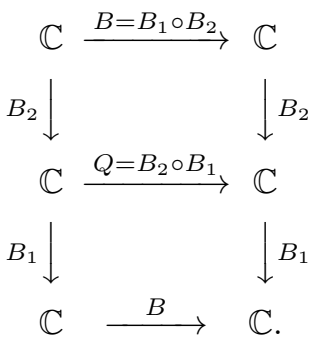

Corollary 8. Let $\hat{B}$ be the dynamical extension of $B=B_{1} \circ B_{2}$ constructed in Corollary 6, then $Q=B_{2} \circ B_{1}$ has a dynamical extension such that there exist two Fuchsian groups $\Gamma(B)$ and $\Gamma(Q)$ and elements $\alpha_{B}, \alpha_{Q}, \beta_{1}$, and $\beta_{2}$ in $\operatorname{PSL}(2, \mathbb{R})$ 
making the following diagrams commutative:
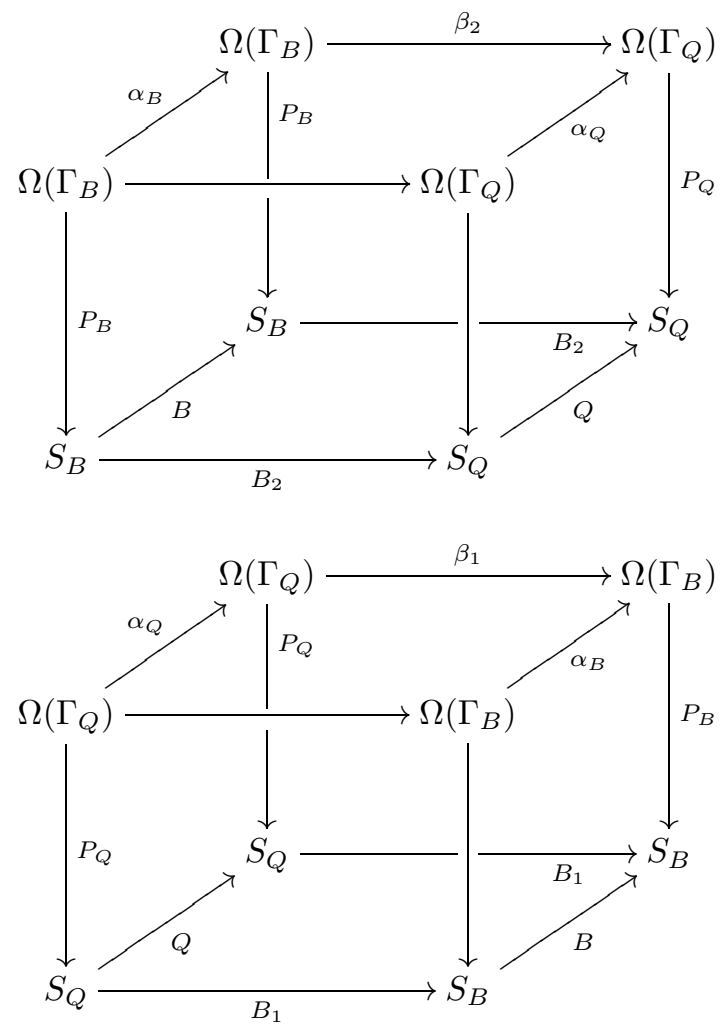

Proof. Note that $B_{2}: S_{B} \rightarrow S_{Q}$ and $B_{1}: S_{Q} \rightarrow S_{B}$ define coverings, hence there are Möbius maps $\beta_{1}: \Omega\left(\Gamma_{Q}\right) \rightarrow \Omega\left(\Gamma_{B}\right)$ and $\beta_{2}: \Omega\left(\Gamma_{B}\right) \rightarrow \Omega\left(\Gamma_{Q}\right)$ which make the diagrams commutative.

As a consequence of Corollary 8 we have the following conclusion.

Proposition 9. Let $B=B_{1} \circ B_{2}$, let $\hat{B}$ be a dynamical extension, then there are Poincaré extensions $\hat{B}_{1}$ and $\hat{B}_{2}$ of $B_{1}$ and $B_{2}$, respectively, such that

$$
\hat{B}=\hat{B}_{1} \circ \hat{B}_{2} \text {. }
$$

Moreover, there exist $\hat{Q}$ a dynamical extension of $Q:=B_{2} \circ B_{1}$ such that

$$
\hat{Q}=\hat{B}_{2} \circ \hat{B}_{1} \text {. }
$$

The following theorem summarizes the results above and shows that the corresponding extensions are geometric.

Theorem 10. Let $B$ be a Blaschke map, then:

(i) The extension constructed in Theorem 4 is geometric.

(ii) The dynamical extension constructed in Corollary 6 is geometric.

(iii) The extensions in Corollary 8 are all geometric.

Each of the extensions in items (i)-(iii) is conformally natural with respect to the group of Möbius transformations that leaves the unit circle invariant. 
Proof. We will show item (i), the proof of the other items apply similar arguments to the extensions constructed in Corollary [6 and Proposition 9, Again, the important feature is that the corresponding surfaces are symmetric with respect to the unit circle. According to Theorem 4 there are manifolds $M_{1}$ and $M_{2}$, Möbius projections $p_{1}$ and $p_{2}$, and a Poincaré extension $\hat{B}$ of $B$ such that the following diagram is commutative such that $\left.p_{i}\right|_{\Omega\left(\Gamma_{i}\right)}=\pi_{i}$ :

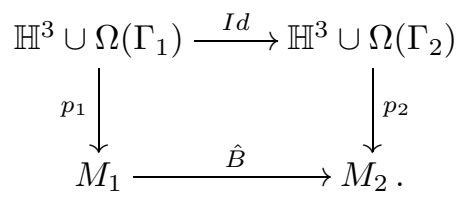

Now, we construct universal coverings $q_{i}$ which maps $\mathbb{H}^{3} \cup \Omega\left(\Gamma_{i}\right)$ in $\overline{\mathbb{B}}^{3}$ and $q_{i}(x)=$ $q_{i}(y)$ if and only if there exist a $\gamma_{i}$ in $\Gamma_{i}$ with $\gamma_{i}(x)=y$, so that

$$
\left.q_{i}\right|_{\Omega\left(\Gamma_{i}\right)}=\left.p_{i}\right|_{\Omega\left(\Gamma_{i}\right)}=\pi_{i}
$$

By Theorem 4 the group $\Gamma_{2}$ acts on the unit disk which belongs to the boundary of $\bar{B}^{3} \cap \mathbb{R}^{2}$ so that $\pi_{2}(\Delta)=S_{2} \cap \Delta$ also belongs to the boundary of $\bar{B}^{3} \cap \mathbb{R}^{2}$. Let $\tau_{\phi}$ be the Möbius rotation, in $\mathbb{R}^{3}$, with respect to $\partial \Delta$ of angle $\phi$. Then

$$
\overline{\mathbb{B}}^{3}=\bigcup_{0 \leq \phi \leq \pi} \tau_{\phi}(\Delta)
$$

and $\tau_{\pi}: \Delta \rightarrow \Delta^{*}$ is the map $z \mapsto 1 / \bar{z}$ in the holomorphic coordinate of $\Delta$. Define $q_{i}(z, \phi)=\left(\tau_{\phi} \circ \pi_{i}(z)\right)$ such that $\tau_{\phi}$ commutes with $\operatorname{Aut}(\Delta) \simeq P S L(2, \mathbb{R})$. Furthermore, $\tau_{\phi}$ commutes with any Möbius map that leaves the unit circle invariant (for instance, $z \mapsto 1 / z)$.

Then $M_{i}=q_{i}\left(\mathbb{H}^{3} \cup \Omega(\Gamma)\right)$ are subsets of $\overline{\mathbb{B}}^{3}$ and the respective Poincaré extension is conformally natural with respect to the group of all Möbius maps that leave the unit circle invariant.

Corollary 11. If in Proposition 9 we have that $B_{1}=B_{2}$, then $\hat{B}_{1}=\hat{B}_{2}$. Using induction, if $B=B_{1}^{n}$, then for every dynamical extension $\hat{B}$ of $B$, there exists a dynamical extension $\hat{B}_{1}$ of $B_{1}$ such that $\hat{B}=\hat{B}_{1}^{n}$.

In the case (i) of Theorem 10, let $Q_{i}: \mathbb{H}^{3} \rightarrow \overline{\mathbb{B}}^{3}$ be other extensions of the projections $\pi_{i}$, then there are continuous maps $h_{i}: M_{i} \rightarrow \overline{\mathbb{B}}^{3}$ such that $Q_{i}=h_{i} \circ q_{i}$. Where $q_{i}$ are the extensions constructed on the proof of Theorem 10.

Let us put $K=Q_{2} \circ Q_{1}^{-1}$ where the composition is defined. If $\hat{B}$ is the geometric extension of $B$ from Theorem 10, then $K \circ h_{2}=h_{1} \circ \hat{B}$.

In the case (ii) of Theorem 10, assume $Q_{i}$ is another extension of $\pi_{i}$. Again, put $K=Q \circ \alpha_{B} \circ Q^{-1}$. If $K$ is a map, then $K$ is semiconjugated to $\hat{B}$.

Theorem 12. Let $E$ be a semigroup of Blaschke maps, then the extension constructed in Theorem 10 defines in $E$ a geometric homomorphic conformally natural extension preserving degree if, and only if, $E$ does not intersect the bi-orbit of $f(z)=z^{n}$ with respect to $\operatorname{Aut}(\Delta)$.

Proof. If the extension is not conformally natural with respect to $\operatorname{PSL}(2, \mathbb{C})$ then there are two elements $B_{1}$ and $B_{2}$ in $S$ with two maps $g_{1}$ and $g_{2}$ in $\operatorname{PSL}(2, \mathbb{C})$ such that $B_{1} \circ g_{1}=g_{2} \circ B_{2}$. Then by Theorem [10, the maps $g_{1}$ and $g_{2}$ cannot leave the unit circle invariant. Then there are two circles $C_{1}=g_{1}\left(\mathbb{S}^{1}\right)$ and $C_{2}=g_{2}\left(\mathbb{S}^{1}\right)$ with 
$B_{1}^{-1}\left(C_{2}\right)=C_{1}$. Let us show that the circles $C_{i}$ do not intersect $\mathbb{S}^{1}$. Assume that there is $x$ in $C_{1} \cap \mathbb{S}^{1}$, then $C_{2}$ intersects $\mathbb{S}^{1}$ in all the preimages of $x$ with respect to $B_{1}$. But this is possible only if the preimage of $x$ under $B_{1}$ is a single critical point, but a Blaschke map cannot have critical points on $\mathbb{S}^{1}$. Therefore, $C_{1}$ and $C_{2}$ cannot intersect $\mathbb{S}^{1}$. Then $C_{1}$ and $\mathbb{S}^{1}$ bound an annulus. By the reflection principle, we have that $B_{1}$ has in the unit disk a critical point of multiplicity $d-1$. Hence either $B_{1}$ or $1 / B_{1}$ belongs to the bi-orbit of $z^{n}$ with respect to $A u t(\Delta)$, but the extension in Theorem 4 is compatible with the map $z \mapsto 1 / z$. Hence the conclusion of the theorem holds. Reciprocally, the extension of Theorem 4 is not conformally natural on the map $z^{n}$.

Corollary 13. Assume $B$ is a Blaschke map of the form $B=g_{1} \circ z^{d} \circ g_{2}$ such that $g_{2}(z) \neq e^{i \alpha} \circ g_{1}^{-1}(z)$, then the extensions constructed on Theorem 10 of $\left\langle B^{n}\right\rangle$ are conformally natural whenever $n \geq 2$.

Proof. If, for $n \geq 2, B^{n}$ does not satisfy the conditions of Theorem 12, then $B^{n}$ should be in the bi-orbit of $z^{d^{n}}$. Hence $B^{n}$ has one critical point and one critical value, this implies that the critical point $x$ of $B$ is fixed. Hence $g_{1} \circ z^{d} \circ g_{2}(x)=x$ then $g_{1}(0)=g_{2}^{-1}(0)$, which implies $g_{2} \circ g_{1}(0)=0$ and $g_{2}=e^{i \alpha} \circ g_{1}^{-1}(z)$.

3.1. Geometric extensions in Hurwitz spaces. Let us recall that a branched covering $f: \overline{\mathbb{C}} \rightarrow \overline{\mathbb{C}}$ of degree $d$ is in general position, if the number of critical points is the same as the number of critical values and equal to $2 d-2$. According to [17] a theorem of Luroth and Clebsch states that:

Lemma 14. Any two branched coverings $f_{i}: \overline{\mathbb{C}} \rightarrow \overline{\mathbb{C}}$ of the same degree in general position are Hurwitz equivalent.

The existence of a Schottky type extension is a property of the whole Hurwitz space as we show in the following.

Lemma 15. Let $B$ be a map with a Schottky type geometric extension and let $R$ be a rational map in $H(B)$, then $R$ also has an extension of Schottky type.

Proof. Let $\Gamma_{1}$ and $\Gamma_{2}$ be the uniformizing groups for $B$. Let $\alpha: \mathbb{C} \rightarrow \mathbb{C}$ be the Möbius map extending $B$. By definition of $H(B)$, there are two quasiconformal maps $f, g$ on the Riemann sphere such that $f \circ B=R \circ g$. Solving the Beltrami equation, we get quasiconformal extensions $\tilde{f}: \mathbb{C} \rightarrow \mathbb{C}$ and $\tilde{g}: \mathbb{C} \rightarrow \mathbb{C}$ of $f$ and $g$, respectively, such that $\beta=\tilde{g}^{-1} \circ \alpha \circ \tilde{f}$ is a Möbius map extending $R$. Let us assume first that $\tilde{f}$ and $\tilde{g}$ have small distortion, then by a theorem in [5, Th. 5] each map, $\tilde{f}$ and $\tilde{g}$, admits a homeomorphic extension, say $\hat{f}$ and $\hat{g}$, to $\mathbb{B}^{3}$ compatible with the groups $\Gamma_{1}$ and $\Gamma_{2}$. Hence we obtain two Kleinian groups $\tilde{\Gamma}_{1}=\hat{f} \circ \Gamma_{1} \circ \hat{f}^{-1}$ and $\tilde{\Gamma}_{2}=\hat{g} \circ \Gamma_{2} \circ \hat{g}^{-1}$ with manifolds $M\left(\tilde{\Gamma}_{1}\right)$ and $M\left(\tilde{\Gamma}_{2}\right)$ that extend the map $R$. To see that this extension is geometric we have to embed each manifold $M\left(\tilde{\Gamma}_{1}\right)$ and $M\left(\tilde{\Gamma}_{2}\right)$ into $\mathbb{B}^{3}$, such that the image of these embeddings are the complement of a finite set of quasigeodesics.

To do so, we use the geometric extension of $B$. We know that $M\left(\Gamma_{1}\right)$ and $M\left(\Gamma_{2}\right)$ are already realized as submanifolds of $\mathbb{B}^{3}$, hence by conjugating $M\left(\Gamma_{1}\right)$ by $\hat{f}$ and $M\left(\Gamma_{2}\right)$ by $\hat{g}$, we obtain the desired embeddings of $M\left(\tilde{\Gamma}_{1}\right)$ and $M\left(\tilde{\Gamma}_{2}\right)$ into $\mathbb{B}^{3}$.

To complete the proof we note that $H(B)$ is connected, so for maps $f$ and $g$ with big distortion, we can use a path on $H(B)$ and extend the maps along the path with small distortion changes. 
Now let us show that there are Schottky type extensions for a large set of rational maps.

Theorem 16. There is an open and everywhere dense subset in $R_{a} t_{d}(\mathbb{C})$ which has a geometric extension of Schottky type of the same degree.

Proof. By Lemma 14, the union of all the Hurwitz spaces of all Blaschke maps of fixed degree is open and everywhere dense in $\operatorname{Rat}_{d}(\mathbb{C})$. Hence Theorem 4 and Lemma 15 imply this theorem.

It follows that structurally stable rational maps have a Schottky type extension. We believe that any rational map has a geometric extension such that the respective manifold belongs to the closure of the Schottky space. Since Hurwitz space of any branched covering of finite degree of the sphere contains a rational map, we conjecture that the closure of the Schottky space of given degree $d$ contains all realizable Hurwitz combinatorics.

3.2. Extensions of exceptional maps. A dynamical Poincaré extension, in general, requires a uniformizable geometric structure which is invariant under a rational map $R$. However, it is rare for a sphere with finitely many punctures to admit a self-covering, with the exception of $z^{n}$. Nevertheless, there are orbifolds $W$, supported on the Riemann sphere, and rational maps $R: W \rightarrow W$, so the map $R$ is an orbifold self-covering. The class of maps $R$ are called exceptional; the reader will find a more detailed discussion of these maps in 13 . In particular, the Euler characteristic $\chi\left(W_{1}\right)$ is zero. Hence $W_{1}$ is a parabolic orbifold, this only occurs when the map $R$ is conjugate to either a Tchebychev type map, a Lattès map or $z \mapsto z^{n}$.

Theorem 17. Let $G$ be a semigroup of rational maps which are self-coverings of a parabolic orbifold $W$ supported on the Riemann sphere. Then there exist a geometric extension satisfying the following conditions:

- For every $g \in G$, the extension $\hat{g}$ has the same degree as $g$.

- Each extension is geometric.

- The set of extensions $\hat{G}$ is a semigroup, and the extension map is a homomorphism from $G$ to $\hat{G}$.

Proof. The proof exploits the fact that the elements in $G$ have known uniformizations. Consider the lattice

$$
L_{\tau}:=\langle z \mapsto z+1, z \mapsto z+\tau: \Im \tau>0\rangle
$$

in the Lattès case, and the lattice

$$
L_{0}:=\langle z \mapsto z+1\rangle
$$

in the case of $z^{n}$ and Tchebychev. According to the classification of Lattès maps given by Milnor (see Theorem 1.1 in [13]), for any holomorphic semigroup of endomorphisms of a flat orbifold structure supported on $\overline{\mathbb{C}}$ there exist $\tau_{0}$ and a Kleinian group $\Gamma_{n}$ such that $\Gamma_{n}$ contains the lattice $L_{\tau_{0}}$ as a subgroup of finite index $n$ and $W$ is equivalent to $\mathbb{C} / \Gamma_{n}$. Here $n$ is necessarily either $2,3,4$ or 6 when $\tau_{0} \neq 0$. In the case where $\tau_{0}=0$, then $n$ is either 1 or 2 .

In terms of the group $\Gamma_{n}, G$ is a semigroup of affine endomorphisms of $\Gamma_{n}$. In each case, each element in $G$ has a simultaneous Poincaré extension on the orbifold $\mathbb{H}^{3} / \Gamma_{n}$. This Poincaré extension satisfies the properties of the theorem. 
Now we discuss the geometric extension of semigroups of the exceptional rational maps mentioned in the theorem above. First, we discuss the case when $n=2$. In this case, $R$ is a holomorphic endomorphism of the orbifold of type $(\overline{\mathbb{C}}, 2,2,2,2)$.

Let us consider the torus $T$ in $\mathbb{C}^{2}$ given in coordinates $\left(z_{1}, z_{2}\right)$ by $\left|z_{1}\right| \leq 1$ and $\left|z_{2}\right|=1$. The core of $T$ is the unit circle $C=\left\{\left(z_{1}, z_{2}\right): z_{1}=0,\left|z_{2}\right|=1\right\}$. The space $T \backslash C$ is uniformized by the Poincaré extension of a lattice $L_{\tau}$ in $\mathbb{H}^{3}$ parallel to the plane, for a suitable choice of $\tau$.

Let $I$ be the involution map

$$
\left(z_{1}, z_{2}\right) \mapsto\left(\bar{z}_{1}, \bar{z}_{2}\right)
$$

The map $I$ acts on the filled torus $T$ as an involution. The quotient $T / I$ gives an orbifold $O$ supported in $\overline{\mathbb{B}}^{3}$ with two ramification lines. Let $\pi: T \rightarrow T / I$ be the orbit projection.

Let us consider the family of endomorphisms $\Psi(l, m, k)$ of $T$ given by the formulae

$$
\left(z_{1}, z_{2}\right) \mapsto\left(z_{1}^{l} z_{2}^{k}, z_{2}^{m}\right),
$$

where $l, m, k$ are integers. In other words, the family $\{\Psi(l, m, k)\}$, for all $l, m, k$, contains the family of all the Poincaré extensions of semigroups of integer multiplications on $T \backslash C$. Then this family commutes with the involution $I$ and generates a semigroup $J$ of endomorphisms of the orbifold $O$.

In contrast with the case $n=2$, when $n>2$ we obtain a finite group $G_{n}$ which acts on the boundary of $T$ as a rotation. The action of $G_{n}$ does not have a continuous extension on the whole of $T$, it is discontinuous on the core. Hence we cannot construct a compact orbifold analogous to $O$. However, there exist an invariant foliation, which implies that, for the cases where $n$ is either 3,4 or 6 , there exist a geometric extension which is isometric to the radial extension. This construction is similar to the construction given in Theorem 3 .

Corollary 18. Let $\hat{R}$ be an element of $J$, then $\pi(C)$ is an interval which is invariant with respect to $\hat{R}$ and the restriction of $\hat{R}$ on $\pi(C)$ is topologically conjugate to a Tchebychev polynomial of degree $k$ restricted on its Julia set. Moreover, there exist a continuous projection $h: \partial O \rightarrow \pi(C)$ so that $h \circ \hat{R}=\hat{R} \circ h$.

Proof. Note that the projection $P: T \rightarrow C$ given by $\left(z_{1}, z_{2}\right) \rightarrow\left(0, z_{2}\right)$ commutes with the maps $\Psi(l, m, k)$ and the involution $I$. Moreover, the restriction of the map $\Psi(l, m, k)$ on $C$ is the power map $z_{2} \mapsto z_{2}^{m}$. The action of $\hat{R}$ restricted on $\pi(C)$ is topologically conjugate to the action of a Tchebychev polynomial on its Julia set. Since the projection $P$ commutes with the action of $I$, then $P$ descends to a projection $h: \partial O \rightarrow \pi(C)$ which satisfies the desired properties.

One would expect that the dynamics of Tchebychev polynomials on its Julia set are obtained by pinching $T$ onto $C$. The projection $P$ defines a foliation on $\partial T$ by circles. Hence, the projection $h$ defines a foliation $F$ on $\partial O$, all leaves in $F$ are topological circles with the exception of two leaves homeomorphic to the interval. In other words, one would think that the foliation $F$ shrinks to a model of the Tchebychev polynomial restricted to its Julia set. So there would be a deformation of the foliation in the boundary $\partial O$ that produces a Tchebychev map. The corollary above suggests an argument to construct such a deformation. So, it is natural to ask: is it true that the closure of the space of quasiconformal deformations of flexible Lattès maps in $\bigcup_{d<\operatorname{deg}(R)} \operatorname{Rat}_{d}(\mathbb{C})$ contains Tchebychev maps? Is it true 
that any point in the boundary of quasiconformal deformations of flexible Lattès map is rigid? Finally, is it true that any point in the boundary has degree strictly smaller than degree of the given Lattès map?

3.3. Non-Galois affine extensions. The main idea of this paper is to transform a rational map to a Möbius morphism. On the sections above, we discussed Galois coverings, which is the uniformizable situation. In this subsection, let us consider non-Galois coverings which also transform rational dynamics into Möbius dynamics.

Simple examples of non-Galois coverings are given by Poincaré functions associated to repelling cycles of $R$. These are functions $f$ satisfying the functional equation $f(\lambda z)=R^{n} \circ f(z)$ for some $n$ and some complex number $\lambda$.

Let us suppose that there exist an extension $\hat{f}$ of $f$ in $\mathbb{H}^{3}$ and consider the multivalued map defined by

$$
K_{f}(z)=\hat{f}\left(\lambda \hat{f}^{-1}(z)\right)
$$

When $K_{f}$ is an ordinary map, we have a dynamical extension. If $f$ is a Galois covering, then we are in the parabolic situation described in the previous subsection. However, is not clear when $K_{f}$ is a map. In this case, we call $\hat{K}_{f}$ a non-Galois extension of $R$ with respect to $f$.

Let $B$ be a Blaschke map, and $R$ a quasiconformal deformation of $B$. Now we show that $R$ has a non-Galois extension with respect to any Poincaré function $f$.

Theorem 19. Let $R$ be quasiconformally conjugate to a Blaschke map. For every Poincaré function of $R$ there exist a non-Galois extension of $R$.

Proof. Let us first consider a Blaschke map $B$. Any Poincaré function of $B$ satisfies

$$
f(\bar{z})=\frac{1}{\overline{f(z)}},
$$

hence $f$ maps the lower half-plane $\mathbb{H}_{-}^{2}$ to the unit disk. Now we are in position to use a similar argument of the proof of Theorem 10 to define an extension $\hat{f}: \mathbb{H}^{3} \rightarrow \mathbb{B}^{3}$ as follows: first identify $\mathbb{H}^{3}$ with the "open book" coordinates $(z, \phi)$ where $z \in \mathbb{H}_{-}^{2}$ and $\phi$ in the interval $(0, \pi)$ and put

$$
\hat{f}(z, \phi)=\tau_{\phi}(f(z))
$$

where $\tau_{\phi}$ is the Möbius rotation of angle $\phi$ in $\mathbb{R}^{3}$ with respect to the unit circle. In this case, from the equation satisfied by a Poincaré function, we have that $K_{f}=\hat{B}^{n}$ for some iterate of $\hat{B}$, where $\hat{B}$ is the dynamical extension constructed in Theorem 10. Let $R=\phi \circ B \circ \phi^{-1}$, then any Poincaré function for $R$ belongs to the Hurwitz space of a suitable Poincaré function of $B$. Now we can apply the arguments in Lemma 15 to finish the proof.

Let us recall that, for every complex affine line $L$ there is a process of hyperbolization $T: L \rightarrow H(L)$ which associates a hyperbolic manifold $H(L)$ to $L$. This hyperbolization process is used in the construction of 3-hyperbolic Lyubich-Minsky laminations in [8]. By this process there is an identification $H(L) \cong \mathbb{C} \times \mathbb{R}_{+}$. Given an affine line $L$, let us assume that we have fixed any such identification.

Now let $L_{1}$ and $L_{2}$ be complex affine lines. Let $F: L_{1} \rightarrow L_{2}$ be any map, then for any $\lambda>0$ there is a family of extensions $\hat{F}_{\lambda}: H\left(L_{1}\right) \rightarrow H\left(L_{2}\right)$ given in coordinates by

$$
\hat{F}_{\lambda}(x, t)=(F(x), \lambda t)
$$


Note that if $F$ is affine then there exists a unique $\lambda_{0}$ such that $F_{\lambda_{0}}$ is the Poincaré extension of $F$ in $H(L)$. Let $q_{i}: L_{i} \rightarrow \mathbb{C}$ be maps for $i=1,2$. Assume we have a polynomial $P$ and an affine map $\gamma: L_{1} \rightarrow L_{2}$ satisfying

$$
P \circ q_{1}=q_{2} \circ \gamma \text {. }
$$

Then for all $\lambda, \omega$ and $\rho$ positive real numbers, we have

$$
\begin{aligned}
\left(q_{2}\right)_{\omega} & \circ \gamma_{\lambda}(x, t)=\left(q_{2}\right)_{\omega}(\gamma(x), \lambda t) \\
& =\left(q_{2}(\gamma(x)), \omega \lambda t\right)=\left(P \circ \pi_{1}(x), \omega \lambda t\right) \\
& =P_{\rho}\left(q_{1}(x), \frac{\omega \lambda}{\rho} t\right)=P_{\rho} \circ\left(q_{1}\right)_{\frac{\omega \lambda}{\rho}}(x, t) .
\end{aligned}
$$

Now let $\lambda_{0}$ be the number such that $\gamma_{\lambda_{0}}$ is the Poincare extension of $\gamma$ in $\mathbb{H}^{3}$ and put $\rho=\lambda_{0}$ in the formula above, then we have

$$
\left(q_{2}\right)_{\omega} \circ \gamma_{\lambda_{0}}=P_{\lambda_{0}} \circ\left(q_{1}\right)_{\omega} \text {. }
$$

Assume that $q_{1}=q_{2}=f$ where $f$ is the Poincaré function of a fixed point with multiplier $\lambda_{0}$, then for every $\omega$ the map $P_{\lambda_{0}}$ is a geometric dynamical extension with the same degree. In this extension, the orbit of every point in $\mathbb{H}^{3}$ converges to infinity. In other words, the Julia set of the extension belongs to $\overline{\mathbb{C}}$. To show that $P_{\lambda_{0}}$ is geometric let $M_{1}$ be the complement in $\mathbb{H}^{3}$ of all vertical lines based on the $P$-preimages of the postcritical set, then $M_{2}$ is the complement in $\mathbb{H}^{3}$ of all vertical lines based on the postcritical set and $P_{\lambda_{0}}: M_{1} \rightarrow M_{2}$. Then $f_{\omega}$ endows $M_{i}$ with incomplete Möbius structures, making $P_{\lambda_{0}}$ geometric.

Another situation is when $\rho=1$, again let $q_{1}=q_{2}=f$ as above. Then $P_{1}$ is a Poincaré extension, with the manifolds $M_{i}$. However, the Möbius structures on $M_{i}$ are different, on $M_{1}$ is given with $f_{\omega}$ and on $M_{2}$ is given by $f_{\lambda_{0} \omega}$. Note that $\rho=1$ gives a homomorphic extension defined on the semigroup of polynomials.

For the reader familiar with the construction of Lyubich-Minksy [8], we note that natural extension of either $P_{\lambda_{0}}$ or $P_{1}$ is equivalent to the 3 -hyperbolic LyubichMinsky lamination.

\section{Product extension}

At least for us, it is very surprising that there is a product structure on $\mathbb{H}^{3}$ which, in a sense, is a "conformal natural" extension of the complex product on $\mathbb{C}$. To construct this product, first let us extend the exponential map $\operatorname{Exp}(z)=e^{z}$. We consider the coordinates $(z, t)$ in $\mathbb{H}^{3}$. Let $h_{\alpha}: \mathbb{H}^{3} \rightarrow \mathbb{H}^{3}$ be the translation given by

$$
(x, y, t) \mapsto(x, y-\alpha, t),
$$

the map $H_{\beta}: \mathbb{H}^{3} \rightarrow \mathbb{H}^{3}$ is the dilation given by

$$
(x, y, t) \mapsto(\beta x, \beta y, \beta t),
$$

and let $p: \triangle \rightarrow \mathbb{H}^{3}$ be the stereographic projection that maps the unit disk $\triangle$ into the unit semisphere in $\mathbb{H}^{3}$.

Write

$$
\Phi(x, 0, t)=p \circ \operatorname{Exp}(x+i t)
$$

and let $V$ be the vertical semiplane over the imaginary line. Then $\Phi$ maps $V$ onto the unit semisphere in $\mathbb{H}^{3}$. Finally, for $w=(x, y, t)$ let

$$
\widehat{\operatorname{Exp}}(w)=H_{e^{-2 \pi y}} \circ \Phi \circ h_{y}^{-1}(w) \text {. }
$$


By construction $\widehat{\operatorname{Exp}}$ maps $\mathbb{H}^{3}$ onto $M$, the complement of the $t$-axis in $\mathbb{H}^{3}$, and is a covering. When $t=0$ the map $\widehat{\operatorname{Exp}}$ coincides with the function Exp. Also, $\widehat{E x p}$ defines a complete Möbius structure $\delta$ on $M$. Any Möbius map that leaves $M$ invariant is Möbius in $\delta$.

Since Exp defines an homomorphism of the additive structure on $\mathbb{C}$ onto the multiplicative structure of $\mathbb{C}^{*}$. Then $\widehat{\operatorname{Exp}}$ gives a multiplication "*" in $M$, which is the pushforward of the additive structure on $\mathbb{H}^{3}$. Indeed, let $a$ and $b$ elements in $M$, and let $a_{1}$ and $b_{1}$ be elements such that $a=\widehat{\operatorname{Exp}}\left(a_{1}\right)$ and $b=\widehat{\operatorname{Exp}}\left(b_{1}\right)$. Then write

$$
a * b=\widehat{\operatorname{Exp}}\left(a_{1}+b_{1}\right) .
$$

This is well defined and $M$ is closed under the $*$ multiplication.

Lemma 20. The multiplicative structure in $M$ extends to a multiplicative structure on $\mathbb{H}^{3}$.

Proof. Let $\|$.$\| be the standard norm in the euclidean space \mathbb{R}^{3}$. Then for every $x$ and $y$ in $\mathbb{H}^{3}$. We have

$$
\|x * y\|=\|x\|\|y\| .
$$

Now, let $x=(0,0, t)$. For any $y \in \mathbb{H}^{3}$ define $y * \tau=(0,0, t\|y\|)$. The restriction of this multiplication to points in the axis $t$ in $\mathbb{H}^{3}$ coincides with the usual multiplication on $\mathbb{R}_{+}$. This definition continuously extends the multiplication $*$ to the whole of $\mathbb{H}^{3}$.

The multiplication in $\mathbb{H}^{3}$ is commutative and associative and has the following properties:

- The multiplication restricted on the boundary is the usual complex multiplication in $\mathbb{C}$.

- Let $\lambda$ be a nonzero complex number then, for any $x \in \mathbb{H}^{3}$, we have $\lambda * x=$ $H_{\lambda}(x)$. Where $H_{\lambda}(x)$ is the Poincaré extension of the map $z \mapsto \lambda z$.

- The unique unit element is $(1,0,0)$. For any $x \neq 0, \infty$ in $\overline{\mathbb{H}}^{3}$ there exists $y$ such that $x * y=y * x=(1,0,0)$ and $y=H(x)$ where $H$ is the Poincaré extension of the map $z \mapsto 1 / z$.

Now we can define an extension of rational maps. Let $R$ be a rational map and consider a decomposition of $R$ as a product of Möbius maps:

$$
R(z)=\prod \gamma_{i}(z)
$$

where $\gamma_{i} \in P S L(2, C)$. Hence for $x \in \overline{\mathbb{H}}^{3}$ we have an extension with respect to the maps $\gamma_{i}$,

$$
\hat{R}(x)=\prod_{*} P\left(\gamma_{i}\right)(x),
$$

where $P\left(\gamma_{i}\right)$ is the Poincaré extension of $\gamma_{i}$ in $\overline{\mathbb{H}}^{3}$. Since the multiplication is commutative then the definition does not depend on the order of the factors $\gamma_{i}$. The following proposition follows from the definition of the product extension.

Proposition 21. The product extension has the following properties:

(1) If $\sigma_{i}$ is the geodesic that connects the pole with the zero of $\gamma_{i}$. Then $\hat{R}\left(\sigma_{i}\right)$ is the t-axis.

(2) The extension $R \mapsto \hat{R}$ is right equivariant with respect to the action of $\operatorname{PSL}(2, \mathbb{C})$. 
(3) Any rational map $R$ has a finite number of decompositions in Möbius factors. Hence there are only finitely many product extensions for each rational map $R$.

There is another extension from $\mathbb{C}$ to $\mathbb{H}^{3}$ which is induced by the product. This extension is given by a monomorphism $\Phi$ from the ring of formal series with the usual multiplication on the complex plane to the ring of formal series with the $*$ multiplication. The map $\Phi$ is continuous on the subring of polynomials. However, it is not clear whether it is still continuous on the subring of absolutely convergent series. Note that this extension is not conformally natural, and is not even right equivariant with respect to the action of $P S L(2, \mathbb{C})$. The map $\Phi$ is not a homomorphism with respect to composition. The biggest semigroup $E$, where $\Phi$ defines a homomorphism with respect to composition, is the generated by $\lambda z^{n}$ for any complex $\lambda$ and $n$ a natural number. Moreover, $\Phi$ on $E$ is conformally natural, geometric and the same degree. In general is not clear when product or ring extensions are geometric. However, numerical calculations of the ring extension of $z^{2}+c$, with $c$ real, suggests that this extension is geometric.

4.1. Some examples of Poincaré extensions of quadratic polynomials. Here we compute some Poincaré extensions. These computations are based on the following formula for the exponential map defined in the previous subsection.

$$
\widehat{\operatorname{Exp}}(x, y, t)=\left(\frac{2 e^{t} \cos (y)}{1+e^{2 t}} e^{x}, \frac{2 e^{t} \sin (y)}{1+e^{2 t}} e^{x}, \frac{e^{2 t}-1}{1+e^{2 t}} e^{x}\right) .
$$

We have the following facts:

- The map $\widehat{E x p}$ is a Poincaré extension of the map $e^{z}$.

- Let $T$ be group generated by the translation $z \mapsto z+2 \pi i$, then the action of $T$ in $\mathbb{C}$ extends an action in $\mathbb{H}^{3}$ generated by the map $(z, t) \mapsto(z+2 \pi i, t)$.

- The orbit space $\mathbb{H}^{3} / T$ is homeomorphic to $B_{L}:=\mathbb{H}^{3} \backslash L$ where $L$ is the $t$-axis.

- There exist a complete hyperbolic Möbius structure on $B_{L}$ so that $\widehat{\operatorname{Exp}}$ : $\mathbb{H}^{3} \rightarrow B_{L}$ defines a Möbius universal covering map.

- The extension from Exp to $\widehat{\operatorname{Exp}}$ is conformally natural.

Let $\mathrm{H}_{2}$ be the Poincaré extension of the Möbius map $z \mapsto 2 z$, hence the map $\hat{Q}=\widehat{E x p} \circ H_{2} \circ \widehat{\operatorname{Exp}}^{-1}: B_{L} \rightarrow B_{L}$ is a Poincaré extension of the map $Q(z)=z^{2}$.

For a circle $C$ in $\partial \mathbb{H}^{3}$, let us define the dome over $C$ as the 2 -sphere with equator $C$ intersected with $\mathbb{H}^{3}$ and will be denoted by $\operatorname{Dome}(C)$.

Using the equations above, we obtain the equation

$$
\hat{Q}(\lambda(X, Y, T))=\lambda^{2}\left(\frac{X^{2}-Y^{2}}{1+T^{2}}, \frac{2 X Y}{1+T^{2}}, \frac{2 T}{1+T^{2}}\right)
$$

with $\lambda \in \mathbb{R}$ and $(X, Y, T) \in$ Dome $\left(\mathbb{S}^{1}\right)$.

If $x=\lambda X, y=\lambda Y$ and $t=\lambda T$, then $\|p\|^{2}=x^{2}+y^{2}+t^{2}=\lambda^{2}$. We have that

$$
\hat{Q}(x, y, t)=\left(\|p\|^{2} \frac{x^{2}-y^{2}}{\|p\|^{2}+t^{2}},\|p\|^{2} \frac{2 x y}{\|p\|^{2}+t^{2}},\|p\|^{2} \frac{2 t\|p\|}{\|p\|^{2}+t^{2}}\right) .
$$

In this case, by the formula above, we have that in fact, $\hat{Q}$ extends to the whole of $\mathbb{H}^{3}$ and $\hat{Q}(0,0, t)=\left(0,0, t^{2}\right)$. Moreover, for every $w$ in $\overline{\mathbb{H}}^{3}$ we have $\hat{Q}(w)=w * w$ where $*$ is the product defined above. The map $\hat{Q}$ commutes with the reflection with respect to the dome. 

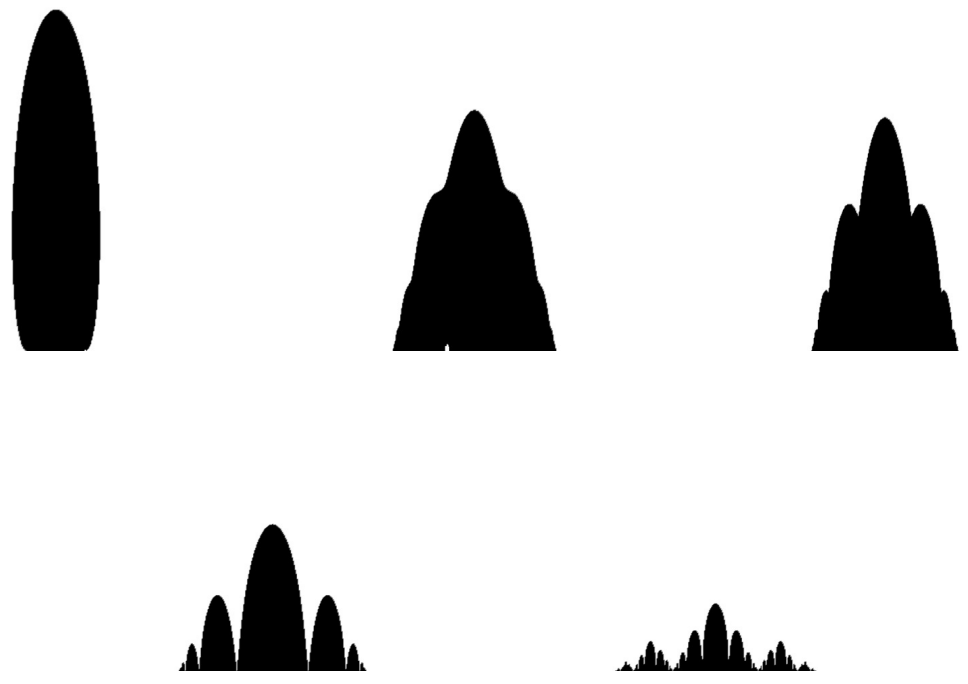

Figure 1. The sets $K\left(\hat{Q}_{c}\right) \cap V_{0}$ for $c=.25,-0.75,-.77,-1,-1.28$ from left to right and top to bottom.

We have the following invariant foliations for the action of $\hat{Q}$ :

(1) Semispheres centered at the origin. Observe that the family of parallel planes of the form $\left(x_{0}, y, t\right)$ is invariant under $H_{2}$. The map $\widehat{\operatorname{Exp}}$ sends this foliation to a foliation of domes over circles centered at the origin. Hence this domes is a foliation invariant under $\hat{Q}$.

(2) Cones centered at the origin: Horizontal planes (horocyclic foliation of $\left.\mathbb{B}^{3}\right)$ of the form $\left(x, y_{0}, z\right)$ are invariant under $H_{2}$, hence their image under $\widehat{\operatorname{Exp}}$ is also invariant. These are cones centered at 0 .

(3) Onion like foliation: Planes of the form $(x, y, k x)$, are invariant under $\mathrm{H}_{2}$. Its image is the onion-like foliation surrounding the vertical axis $(0,0, t)$. This is a book decomposition, here the binding of the book is the unit circle in the boundary plane.

Let $T_{c}(x, y, t)=(x+\operatorname{Re}(c), y+\operatorname{Im}(c), t)$ be the Poincaré extension of the map $z \rightarrow z+c$ and $L_{c}$ the vertical line over $c$ in $\mathbb{H}^{3}$, and let $B_{L_{c}}=\mathbb{H}^{3} \backslash L_{c}$. If $Q_{c}(z)=z^{2}+c$, then we have an extension $\hat{Q}$ of $Q_{c}$ depicted in the following commutative diagram:

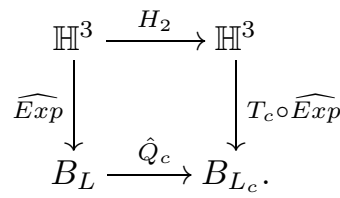

The diagram implies that $\hat{Q}_{c}=T_{c} \circ \hat{Q}$. In this case, the line $L$ is also the critical line but has complicated dynamics. Let us define $K\left(\hat{Q}_{c}\right):=$ spatial filled Julia set of $\hat{Q}_{c}$, the set of $(x, y, t)$ such that $\hat{Q}_{c}^{n}(x, y, t)$ does not tend to $\infty$ as $n \rightarrow \infty$.

Using similar arguments as in the one-dimensional case, one can show that $K\left(\hat{Q}_{c}\right)$ is always bounded in $\mathbb{H}^{3}$. Also, for parameters $c$ with $|c|$ large enough, the critical 
line converges to infinity. If $V_{0}$ denotes the semiplane $\left\{(x, y, t) \in \mathbb{H}^{3}: y=0\right\}$. The section $K\left(\hat{Q}_{c}\right) \cap V_{0}$ is a bi-dimensional set that we have illustrated in Figure 1 for different values of $c$.

\section{REMARKS AND CONCLUSIONS}

As mentioned in the introduction, there are some constructions in the literature of extensions of rational maps into endomorphisms of $\mathbb{H}^{3}$. Most of these extensions are based on the barycentric construction suggested by Choquet's Theorem. Let us briefly describe the barycentric extension. Let $\overline{\mathbb{B}}^{3}$ be the closed unit ball in $\mathbb{R}^{3}$. Let $\mathcal{M}$ be the space of probability measures on $\partial \mathbb{B}^{3}$. Then, for every $\mu$ in $\mathcal{M}$ the barycenter of $\mu$ is the unique point $x$ such that for every functional $L$ on $\mathbb{R}^{3}$, the following equation holds:

$$
L(x)=\int_{\partial \mathbb{B}^{3}} L(y) d \mu(y) .
$$

We define $\operatorname{Bar}(\mu)=x$, by Choquet's theorem (See [15], page 48) the map Bar sends $\mathcal{M}$ onto $\overline{\mathbb{B}}^{3}$. The semigroup $\operatorname{Rat}(\mathbb{C})$ acts in $\mathcal{M}$ by a pushforward, for every $R$ in $\operatorname{Rat}(\mathbb{C})$ we denote by $R \mu=R_{*}(\mu)$ the pushforward of $\mu$ by $R$. For every point $x \in \mathbb{B}^{3}$, let $\nu_{x}$ be the visual measure based on $x$ we define the barycentric extension of $R$ by

$$
\hat{R}(x)=\operatorname{Bar}\left(R \nu_{x}\right) .
$$

Then the barycentric extension is visual as is proved in 12. If instead we use the conformal barycenter we obtain a conformally natural extension as is proved in 14. It is very difficult to get any geometric information of these extensions. For instance, it is not clear that these extensions define a branched covering of the same degree from $\overline{\mathbb{H}}^{3}$ to $\overline{\mathbb{H}}^{3}$.

The following proposition was already mentioned in [7. We include the proof for completeness.

Proposition 22. Let $R$ be a rational map, then all conformally natural extensions of $R$ are homotopic, with a homotopy that consists of conformally natural extensions of $R$.

Proof. Let $\hat{Q}$ and $\hat{S}$ be extensions of a rational map $R$, and let $x \in \mathbb{H}^{3}$. For $\lambda$ in $[0,1]$, let $E_{\lambda}(x)$ be the point along the geodesic from $\hat{Q}(x)$ to $\hat{S}(x)$, which is at distance $\lambda d(\hat{Q}(x), \hat{S}(x))$. Since for every $x \in \partial \mathbb{H}^{3}, \hat{Q}(x)=\hat{S}(x)=R(x)$. The map $E_{\lambda}(x)$ extends to $\partial \mathbb{H}^{3}$ as an extension of $R$. If the maps $\hat{Q}$ and $\hat{S}$ are either right equivariant with respect to the action of $P S L(2, \mathbb{C})$, conformally natural or Poincaré, the map $E_{\lambda}(x)$ also holds the same property.

It follows that if for a given rational map $R$ there are two right equivariants with respect to the action of $\operatorname{PSL}(2, \mathbb{C}$ ) (or conformally natural) extensions, then there are uncountably many right equivariants with respect to the action of $P S L(2, \mathbb{C})$ (or conformally natural) extensions. This situation contrasts with the product extensions which are only finitely many.

The extension discussed in [9] is uniformly quasiregular dynamical and has the same degree as the starting map $R$; moreover, it can be shown that it is geometric. However, for most rational maps these extensions do not exist 9 . 
Another aspect of our geometric construction is about Maskit surgery on the respective Möbius manifolds. A rational map $R: S_{1} \rightarrow S_{2}$ is modeled with two groups $\Gamma_{1}<\Gamma_{2}$. Let us assume that $\Gamma_{2}=\left\langle\gamma_{1}, \ldots, \gamma_{n}\right\rangle$. For $1<k<n G=$ $\left\langle\gamma_{1}, \ldots, \gamma_{k}\right\rangle$ and $H=\left\langle\gamma_{k+1}, \ldots, \gamma_{n}\right\rangle$, and consider the intersections $G_{i}=\Gamma_{i} \cap G$ and $H_{i}=\Gamma_{i} \cap H$. Then $G_{1}$ and $H_{1}$ are subgroups of finite index in $G_{2}$ and $H_{2}$ respectively. This construction defines two rational maps $R_{G}$ and $R_{H}$ associated to groups $G_{i}$ and $H_{i}$ respectively. It is possible to define analogously amalgamated products and HNN-extensions.

\section{ACKNowledgments}

The authors would like to thank M. Kapovich for useful comments and suggestions on an early draft of this paper. Also, we would like to thank the referee for carefully reading and helpful comments to correct the initial manuscript.

\section{REFERENCES}

[1] W. Abikoff, Conformal barycenters and the Douady-Earle extension-a discrete dynamical approach, J. Anal. Math. 86 (2002), 221-234, DOI 10.1007/BF02786649. MR.1894482 (2005d:37099)

[2] L. Bers, Simultaneous uniformization, Bull. Amer. Math. Soc. 66 (1960), 94-97. MR0111834 (22 \#2694)

[3] L. Bers, On boundaries of Teichmüller spaces and on Kleinian groups. I, Ann. of Math. (2) 91 (1970), 570-600. MR0297992 (45 \#7044)

[4] G. Besson, G. Courtois, and S. Gallot, Lemme de Schwarz réel et applications géométriques (French), Acta Math. 183 (1999), no. 2, 145-169, DOI 10.1007/BF02392826. MR.1738042 (2001c:53038)

[5] A. Douady and C. J. Earle, Conformally natural extension of homeomorphisms of the circle, Acta Math. 157 (1986), no. 1-2, 23-48, DOI 10.1007/BF02392590. MR857678 (87j:30041)

[6] R. S. Kulkarni and U. Pinkall, A canonical metric for Möbius structures and its applications, Math. Z. 216 (1994), no. 1, 89-129, DOI 10.1007/BF02572311. MR1273468 (95b:53017)

[7] S. Li, L. Liu, and W. Su, A family of conformally natural extensions of homeomorphisms of the circle, Complex Var. Elliptic Equ. 53 (2008), no. 5, 435-443, DOI 10.1080/17476930701709104. MR2410342 (2010a:30035)

[8] M. Lyubich and Y. Minsky, Laminations in holomorphic dynamics, J. Differential Geom. 47 (1997), no. 1, 17-94. MR1601430 (98k:58191)

[9] G. J. Martin, Extending rational maps, Conform. Geom. Dyn. 8 (2004), 158-166 (electronic), DOI 10.1090/S1088-4173-04-00115-8. MR2122524 (2006b:37084)

[10] B. Maskit, On boundaries of Teichmüller spaces and on Kleinian groups. II, Ann. of Math. (2) 91 (1970), 607-639. MR0297993 (45 \#7045)

[11] C. McMullen, Cusps are dense, Ann. of Math. (2) 133 (1991), no. 1, 217-247, DOI 10.2307/2944328. MR:1087348 (91m:30058)

[12] C. T. McMullen, Renormalization and 3-manifolds which fiber over the circle, Annals of Mathematics Studies, vol. 142, Princeton University Press, Princeton, NJ, 1996. MR 1401347 (97f:57022)

[13] J. Milnor, On Lattès maps, Dynamics on the Riemann sphere, Eur. Math. Soc., Zürich, 2006, pp. 9-43, DOI 10.4171/011-1/1. MR2348953(2009h:37090)

[14] C. Petersen, Conformally Natural extensions revisited, Arxiv Math, http://arxiv.org/abs/ 1102.1470, (2011).

[15] R. R. Phelps, Lectures on Choquet's theorem, 2nd ed., Lecture Notes in Mathematics, vol. 1757, Springer-Verlag, Berlin, 2001. MR1835574 (2002k:46001)

[16] P. Scott, The geometries of 3-manifolds, Bull. London Math. Soc. 15 (1983), no. 5, 401-487, DOI 10.1112/blms/15.5.401. MR705527 (84m:57009)

[17] R. Skora, Maps between surfaces, Trans. Amer. Math. Soc. 291 (1985), no. 2, 669-679, DOI 10.2307/2000104. MR800257(87c:57001) 
Universidad Nacional Autonoma de Mexico, Unidad Cuernavaca del Instituto de Matematicas, Universidad s/n, Col Lomas de Chamilpa, 62210 Cuernavaca, Mexico

E-mail address: carloscabrerao@im.unam.mx

Universidad Nacional Autonoma de Mexico, Unidad Cuernavaca del Instituto de Matematicas, Universidad s/n, Col Lomas de Chamilpa, 62210 Cuernavaca, Mexico

E-mail address: makienko@matcuer.unam.mx

Universidad Nacional Autonoma de Mexico, Facultad de Ciencias, Av. Universidad 3000, 04510 Mexico, Mexico

E-mail address: gsl@dinamical.fciencias.unam.mx 